\title{
NGHIÊN CỨU CÁC YẾU TỐ ẢNH HƯởNG ĐẾN HÀNH VI MUA SĂM TRỤC TUYẾN TRÊN FACEBOOK CỦA NGƯỜ TIÊU DÙNG TẠI THÀNH PHỐ HỒ CHÍ MINH
}

\author{
NGÔ XUÂN NIỆM, BÙI VĂN QUANG \\ Truờng Đại học Công nghiệp thành phố Hồ Chí Minh \\ bvquang09@gmail.com
}

Tóm tắt. Ngày nay với sự phát triển mạnh mẽ của mạng internet và tỉ lệ người dùng các thiết bị thông minh như máy tính bảng, điện thoại thì đã kéo theo sự phát triển của mạng xã hội, trong đó điển hình nhất là Facebook. Mạng xã hội Facebook là một nền tảng của thương mại điện tử. Điều này cho phép khách hàng tham gia một cách tích cực vào thị trường và tiếp cận với một số khối lượng lớn hàng hóa, dịch vụ trong một thời gian ngắn. Các tác động to lớn từ sự phát triển mạng xã hội Facebook không chỉ ảnh hưởng đến hoạt động của các doanh nghiệp mà còn thay đổi cách thức hành xử người tiêu dùng. Mục tiêu của đề tài là nghiên cứu các yếu tố ảnh hưởng đến hành vi mua sắm trực tuyến trên Facebook của người tiêu dùng tại TP.HCM. Nghiên cứu đề xuất 6 yếu tố: Niềm tin đối với sản phẩm, niềm tin về những ảnh hưởng của môi trường xã hội, chính sách hoàn trả, uy tín người bán, sự thuận tiện, livestream-truyền tải trực tuyến.

Với việc đánh giá độ tin cậy của thang đo Cronbach's Alpha, phân tích nhân tố khám phá EFA và kiểm định các giả thuyết đặt ra. Kết quả cho thấy trong 6 yếu tố được đưa vào mô hình thì có đủ 6 yếu tố tác động đến hành vi mua sắm

Từ khóa: hành vi mua sắm trực tuyến, facebook, người tiêu dùng, quần áo thời trang, mạng xã hội.

\section{FACTORS AFFECIG ONLINE SHOPPING BEHAVIOR ON FACEBOOK OF THE CUSTOMERS IN HO CHI MINH CITY}

\begin{abstract}
Today, with the growth of the Internet and the proportion of people using smart devices such as tablets and phones, it has led to the development of social networking, the most typical of which is Facebook. Facebook social networking is a platform of e-commerce. This allows customers to actively participate in the market and access a large number of goods and services in a short period of time. The tremendous impact of Facebook's social networking has not only affected business operations but also changed the way consumers behaved.This research proposes 6 factors effecting online shopping behavior on Facebook of the customers in Ho Chi Minh city. These are trust in the product, trust in community effects, refund policy, seller's credibility, the convenience, livestream-online marketing.

By evaluating the reliability of the Cronbach's Alpha Measures Scale, Exploratory Factor Analysis (EFA) and verifying the assumption. The results shows in 6 factors mentioned, all of them cause impact on customer behavior
\end{abstract}

Keywords: online shopping behavior, Facebook, consumer, clothing fashion, social network

\section{GIỚI THIỆ}

Với sự tiến bộ của công nghệ đã cho ra đời của nhiều mạng xã hội trong đó điển hình nhất là Facebook. Các tác động to lớn từ sự phát triển mạng xã hội Facebook không chỉ ảnh hưởng đến hoạt động của các doanh nghiệp mà còn thay đổi cách thức hành xử người tiêu dùng. Nhận thấy được Facebook là một môi trường kinh doanh mua sắm đầy tiềm năng, rất nhiều doanh nghiệp đã quảng bá sản phẩm của mình trên Facebook.Đông Nam Á nằm trong Top 10 quốc gia có đông người dùng Facebook nhất. Trong đó, Việt Nam xếp thứ 7 với 64 triệu người dùng, chiếm 3\% tổng số tài khoản Facebook toàn cầu. Còn nếu xếp hạng theo cấp thành phố thì TP.HCM đứng thứ 10 trong nhóm 10 thành phố có số người dùng Facebook đông đảo nhất thế giới với 14 triệu tài khoản hoạt động, chiếm $0,6 \%$ tổng số người dùng mạng xã hội này.

Mặc dù trên thế giới và trong nước có rất nhiều đề tài nghiên cứu về các yếu tố hành vi ảnh hưởng đến mua sắm trực tuyến của người tiêu dùng nhưng FB là một mạng xã hội hoàn toàn khác biệt, nó ngày càng 
đổi mới liên tục, xu hướng mua sắm trên mạng xã hội Facebook ngày càng tăng cao mang lại rất nhiều lợi nhuận cũng như tiết kiệm được chi phí cho các tổ chức, doanh nghiệp, cá nhân. Do đó đề tài "Nghiên cứu các yếu tố ảnh hưởng đến hành vi mua sắm trực tuyến trên Facebook của người dùng tiêu dùng tại thành phố HCM" là cần thiết để tìm ra các yếu tố ảnh hưởng đến hành vi mua sắm trực tuyến của người tiêu dung và từ đó có những đề xuất giúp phát triển kênh mua sắm trực tuyến trên Facebook hiệu quả, đáp ứng nhu cầu khách hàng và khai thác lợi thế tối đa của Facebook cho hoạt động kinh doanh của doanh nghiệp, tổ chức, cá nhân trong nhóm ngành hàng quần áo thời trang.

\section{CƠ SỞ LÍ THUYẾT VÀ MÔ HİNH NGHIÊN CÚU}

\subsection{Các khái niệm và cơ sở lý thuyết}

Người tiêu dùng: Theo Philip Kotler, người tiêu dùng gồm các cá nhân hoặc hộ gia đình mua hàng hóa và dịch vụ nhằm thỏa mãn nhu cầu cá nhân. Họ cũng là người tiêu dùng sản phẩm cuối cùng, họ mua và trực tiếp sử dụng sản phẩm, không sử dụng sản phẩm đã mua vào bất kỳ mục đích bán lại.

Hành vi người tiêu dùng: Tổng hợp những phản ứng của người tiêu dùng bởi các yếu tố như những kích thích bên ngoài và diễn biến quá trình tâm lý bên trong thông qua quá trình quyết định lựa chọn hàng hóa hoặc dịch vụ.

Hành vi mua sắm trực tuyến của người tiêu dùng bao gồm những phản ứng và hành động có liên quan tới việc mua sắm sản phẩm hay sử dụng dịch vụ thông qua internet, các phương tiện điện tử.

Facebook là mạng xã hội hàng đầu trên thế giới hiện nay và đứng số 1 tại Việt Nam. Mạng xã hội có nghĩa là nơi kết nối mọi người ở mọi nơi lại với nhau. Nơi các thành viên tương tác với nhau như chat, tải hình ảnh lên, bình luận... Facebook xoá tan mọi khoảng cách địa lý giống như mạng Internet.

\subsection{Một số nghiên cúu có liên quan}

\section{Dahiya Richa (2012)}

Sử dụng phương pháp nghiên cứu định tính và định lượng để nghiên cứu tác động của các yếu tố nhân khẩu học của người tiêu dùng đến mua sắm trực tuyến như sự hài lòng với mua sắm trực tuyến, dự định mua trong tương lai, tần suất mua sắm trực tuyến, số lượng các mặt hàng mua, và tổng chi tiêu cho mua sắm trực tuyến. Các dữ liệu được thu thập thông qua bảng câu hỏi với kích thước mẫu là 580 người trả lời từ Delhi, Mumbai, Chennai, Hyderabad và Bangalore. Kết quả nghiên cứu cho thấy rằng mua sắm trực tuyến ở Ân Độ bị ảnh hưởng đáng kể bởi các yếu tố nhân khẩu học khác nhau như tuổi tác, giới tính, tình trạng hôn nhân, quy mô gia đình và thu nhập.

\section{Na Li và Ping Zhang}

Nghiên cứu: "thái độ hành vi khách hàng trong mua sắm trực tuyến" của $\mathrm{Na} L i$ và Ping Zhang đã đưa ra và lý giải mô hình hành vi khách hàng trong mua sắm trực tuyến. Nghiên cứu cũng đưa ra các biến phụ thuộc, biển độc lập thường sử dụng trong các nghiên cứu về hành vi mua sắm trực tuyến của người tiêu dùng.

\section{MU Sultan \& M Uddin (2011)}

Nghiên cứu về thái độ của khách hàng đến mua sắm trực tuyến tại Gotland, cụ thể là sinh viên Đại học Gotland, các thư viện trường Đại học và thư viện công cộng tại Gotland. Kết quả nghiên cứu cho rằng yếu tố tác động mạnh nhất đến hành vi mua sắm trực tuyến là thiết kế Website/ đặc trưng, kế đến là sự thuận tiện, sự tiết kiệm thời gian và cuối cùng là sự bảo mật. Ngoài ra nghiên cứu cũng cho thấy ngoài nhưng yếu tố kể trên thì có một số yếu tố như giá, chiết khấu, thông tin phản hồi từ những khách hàng trước đó và chất lượng của sản phẩm cũng ảnh hưởng đến dự định hành vi mua sắm trực tuyến.

Theo AhmadRezaAsadollahi et al. (2012)

"Phân tích các yếu tố ảnh hưởng đến dự định hành vi mua sắm trực tuyến của người tiêu dùng có thể là một trong những vấn đề quan trọng nhất của thương mại điện tử và lĩnh vực tiếp thị”. Trong nghiên cứu này thì tác giả đã sử dụng một mô hình kiểm tra sự ảnh hưởng của rủi ro nhận thức, cơ sở vật chất và chính sách hoàn trả về thái độ của người tiêu dùng với hành vi mua sắm trực tuyến và các quy chuẩn chủ quan, cảm nhận về sự tự chủ trong hành vi, lĩnh vực sáng tạo cụ thể và thái độ về hành vi mua sắm trực tuyến. Kết quả của nghiên cứu chỉ ra các rủi ro không giao hàng và về rủi ro tài chính có tác động tiêu cực đến thái độ đối với mua sắm trực tuyến của người tiêu dùng.

\subsection{Các giả thuyết và mô hình nghiên cứu}

\section{Niềm tin đối với sản phẩm}

Niềm tin sản phẩm: là các niềm tin về chất lượng của sản phẩm. Sản phẩm có bị hư hại, kém chất lượng, có đúng như những gì mà doanh nghiệp đã quảng cáo hay không. Sản phẩm có mang lại lợi ích mà người 
tiêu dùng mong muốn hay không. Xét cho cùng các mặt hàng được bán trên Facebook vẫn chủ yếu là bằng hình ảnh, hình ảnh này đã được qua chỉnh sửa để tăng tính thẩm mỹ để dễ thu hút được người tiêu dùng. Đối với những sản phẩm như vậy thì người tiêu dùng khó có thể mà đánh giá được chất lượng, xác định được nguồn gốc xuất xứ sản phẩm. Tác giả đưa ra giả thuyết khi mua hàng trên Facebook, niềm tin về thuộc tính sản phẩm càng cao thì càng lớn tới hành vi mua sắm trực tuyến và ảnh hưởng cùng chiều $(+)$ với hành vi mua sắm trực tuyến

H1: Niềm tin đối với sản phẩm ảnh hưởng cùng chiều (+) với hành vi mua sắm trục tuyến

Niềm tin về những ảnh hưởng của môi trường xã hội

Đặc điểm của bán hàng trên Facebook là dưới mỗi bài bán hàng đều có những phản hồi của khách hàng. Người tiêu dùng có thể xem qua những phản hồi này để đánh giá về uy tín của người bán hàng trên Facebook. Nó có ảnh hưởng rất lớn tới hành vi mua sắm, khi có những phản hồi tốt thì người tiêu dùng sẽ cảm thấy an tâm hơn về sản phẩm của doanh nghiệp và ngược lại. Đặc biệt là khi những sản phẩm đấy đã được người thân, bạn bè, gia đình, đồng nghiệp từng mua thì đó là những phản hồi tốt nhất ảnh hưởng trực tiếp tới hành vi mua sắm trên Facebook của người tiêu dùng. Các nghiên cứu trước từ việc áp dụng và mở rộng các mô hình lý thuyết hành động hợp lý đã cho thấy các đánh giá từ gia đình, họ hàng, bạn bè có ảnh hưởng đến hành vi mua sắm của người tiêu dùng ( Ribbink \& Van, 2004). Những người chưa mua hoặc do dự sẽ có xu hướng đọc những bài nhận xét của những người đã mua hàng để đưa ra quyết định của mình( Ellison, Steinfield. \& Lampe, 2007).

H2: Niềm tin về nhũng ảnh hưởng của môi truờng xã hội tác động cùng chiều (+) với hành vi mua sắm trục tuyến của người tiêu dùng

\section{Chính sách hoàn trả}

Hoàn trả là một trong những điều mà người tiêu dùng cực kì quan tâm và lo ngại nhất khi mua hàng trực tuyến trên Facebook. Mức độ của sự không chắc chắn các rủi ro gặp phải trong suốt quá trình mua sắm trực tuyến có ảnh hưởng mạnh mẽ tới hành vi người tiêu dùng(Bhatnagar và cộng sự ,2000). Ngoài ra thì theo nghiên cứu của AhmadRezaAsadollahi et al. (2012), kết quả cho thấy rủi ro về chính sách hoàn trả ảnh hưởng tiêu cực đến thái độ đối với mua sắm trực tuyến

H3: Chính sách hoàn trả tác động cùng chiều (+) với hành vi mua sắm trục tuyến trên FB của người tiêu dùng.

\section{Uy tín người bán}

Dựa trên một số kết quả nghiên cứu trước đây đã cho thấy sự tin tưởng là một yếu tố quan trọng trong mối quan hệ giữa thái độ và dự định hành vi mua sắm trực tuyến(Shyh-Hwang Lee \& Hoang Thi Bich Ngoc (2010)).Trong bối cảnh mua sắm trực tuyến, khái niệm niềm tin được hiểu là độ sẵn lòng chấp nhận tình trạng có thể bị rủi ro( bị tổn thương) của người tiêu dùng từ việc giao dịch trực tuyến (McKingiht, Choudhury, \& Kacmar, 2002). Sự tin tưởng là cốt lõi của các cuộc mua bán trên thị trường đặc biệt là trong các mua bán trực tuyến bởi vì người mua không thể tiếp xúc với người bán cũng như sản phẩm họ định mua.Vì vậy mà người bán phải có đủ uy tín và danh tiếng tốt

H4: Uy tín người bán tác động cùng chiều (+) với hành vi mua sắm trục tuyến của người tiêu dùng.

\section{Sự thuận tiện}

Nhận thức về sự thuận tiện: là một tập hợp các lợi ích, tiện ích, sự thuận tiện và các giá trị mà nhà cung ứng mang đến cho người tiêu dùng nhằm khơi dậy sự yêu thích và sự quan tâm của người khác. ( Porat, Liss, Tractinsky 2007). Người tiêu dùng có thoải mái lựa chọn những sản phẩm mình mong muốn một cách thoải mái và ngày nay với sự phát triển thì nếu ở bất kì ở đâu chỉ với smartphone hay phương tiện điện tử thì người tiêu dùng cũng có thể mua sắm, lựa chọn hàng hóa, nắm bắt phương thức đặt hàng một cách dễ dàng H5: Sự thuận tiện tác động cùng chiều (+) với hành vi mua sắm trực tuyến của người tiêu dùng.

\section{Livestream- Truyền tải trực tuyến}

Xu hướng bán hàng trực tuyến hiện tại là video bán hàng. Ngày nay càng nhiều doanh nghiệp, tổ chức, cá nhân sử dụng phương thức này bởi vì nhiều lý do sau: Video thể hiện tính chân thực của sản phẩm mình bán. Sản phẩm được thể hiện từ nhiều góc độ và ánh sang khác nhau, sản phẩm được đặt trong môi trường sống thật, người xem còn được tương tác với người bán trong video cho nên người tiêu dùng ít khi cảm thấy không hài lòng khi nhận được sản phẩm bởi vì chúng gần như giống hệt trong khi Livestream

H6: Livestream-truyền tải trục tuyến tác động cùng chiều (+) với hành vi mua sắm trục tuyến của người tiêu dùng. 
Tác giả đã đề xuất mô hình nghiên cứu như sau:

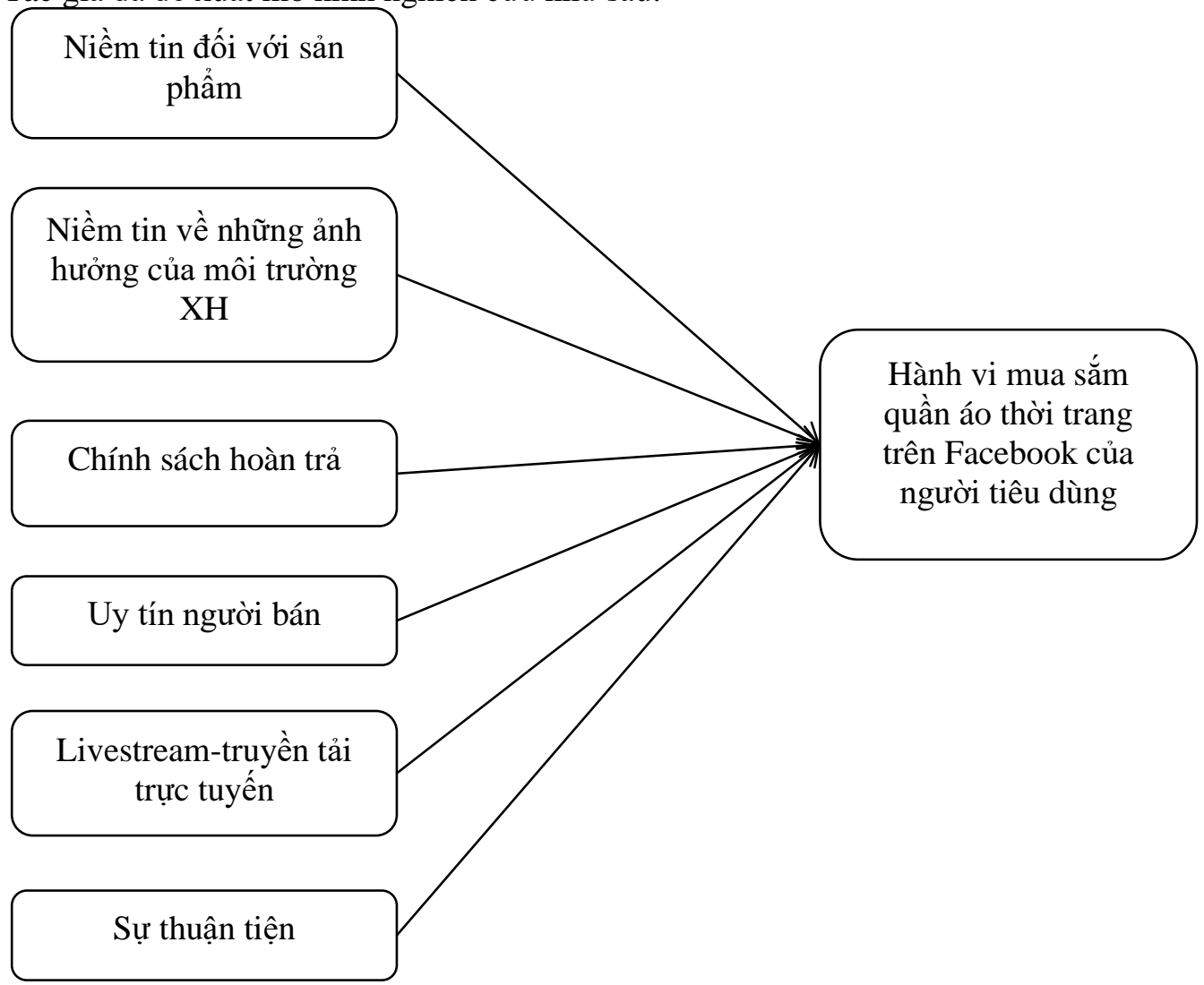

Hình 1: Mô hình nghiên cứu

\section{PHƯƠNG PHÁP NGHIÊN CÚU:}

Mô hình và các giả thuyết được kiểm định dựa trên bộ dữ liệu có kích thước 300 phần tử mẫu. Mẫu được thu nhập qua cuộc khảo sát theo phương pháp lấy mẫu thuận tiện tại Tp. Hồ Chí Minh. Đối tượng khảo sát là người tiêu dùng đã từng mua hàng trên Facebook trong lĩnh vực quần áo thời trang độ tuổi từ 18 - 34 tuổi. Phương pháp tiếp cận là phỏng vấn trực tiếp tại nơi làm việc, học tập hoặc nơi thuận tiện, gửi email khảo sát.

Sau khi bảng câu hỏi định tính được hình thành thì phát ngẫu nhiên cho các quản lý bán hàng trên Facebook, chủ shop quần áo đang bán hàng trên Facebook để kiểm tra lại về tính ý nghĩa của câu hỏi. Đồng thời, tác giả cũng xin ý kiến của giảng viên của khoa Quản trị kinh doanh của trường đại học công nghiệp TPHCM để điều chỉnh về bảng câu hỏi và hoàn thành bảng câu hỏi chính thức. Các đối tượng được phỏng vấn với những đặc điểm khác nhau sẽ cung cấp các thông tin đa chiều và đầy đủ cho nội dung nghiên cứu nhằm đảm bảo được mục tiêu đề ra. Các đối tượng được phỏng vấn trực tiếp. Với mục tiêu đề ra thì các cuộc phỏng vấn đã tìm ra được những yếu tố mới, loại bỏ những yếu tố không còn phù hợp với tình hình thực tế hiện tại

\section{KẾT QUẢ NGHIÊN CÚU \\ 4.1 Mô tả mẫu}

Đặc điểm nhân khẩu học của 300 mẫu nghiên cứu như sau: Đa số họ ở lứa tuổi thanh niên (nhóm tuổi từ 25-30 tuổi, chiếm 183 người trên tổng 300 mẫu, tỉ lệ $61 \%$ và tỷ lệ nữ giới mua hàng nhiều hơn nam giới. Trong đó thì trình độ người tiêu dùng mua sắm FB nhiều nhất thuộc nhóm cao đẳng, đại học chiếm tới $75 \%$ với 225 quan sát. Đa số họ là nhân viên văn phòng với tỷ lệ 58, $3 \%$. 
Bảng 1: Thống kê mô tả mẫu

\begin{tabular}{|c|c|c|c|}
\hline \multirow{2}{*}{\multicolumn{2}{|c|}{ Thông tin mẫu }} & \multirow{3}{*}{\begin{tabular}{|l|} 
Số lượng \\
88 \\
\end{tabular}} & \multirow{3}{*}{$\begin{array}{l}\text { Tỉ lệ } \\
29,3 \% \\
\end{array}$} \\
\hline & & & \\
\hline \multirow{3}{*}{ Độ tuổi } & Dưới 25 & & \\
\hline & 25 đến 30 & 183 & $61 \%$ \\
\hline & Trên 30 tuổi & 29 & $9,7 \%$ \\
\hline \multirow{2}{*}{ Giới tính } & Nam & 72 & $24 \%$ \\
\hline & Nũ & 228 & $76 \%$ \\
\hline \multirow{4}{*}{ Nghề nghiệp } & Sinh viên & 43 & $14,3 \%$ \\
\hline & Nhân viên văn phòng & 175 & $58,3 \%$ \\
\hline & Quản lí & 45 & $15 \%$ \\
\hline & khác & 37 & $12,3 \%$ \\
\hline \multirow{5}{*}{ Trình độ } & Phồ thông & 12 & $4 \%$ \\
\hline & Trung học & 335 & $11 \%$ \\
\hline & Cao đẳng đại học & 225 & $75 \%$ \\
\hline & Trên đại học & 28 & $9,3 \%$ \\
\hline & Khác & 2 & $0,7 \%$ \\
\hline \multirow{6}{*}{ Thu nhập } & <3 triệu & 31 & $10,3 \%$ \\
\hline & 3 đến 5 triệu & 63 & $21 \%$ \\
\hline & 6 đến 8 triệu & 93 & $31 \%$ \\
\hline & 8 đến 10 triệu & 61 & $20,3 \%$ \\
\hline & 10 đến 15 triệu & 31 & $10,3 \%$ \\
\hline & Trên 15 triệu & 21 & $7 \%$ \\
\hline \multirow{4}{*}{ Tần suất mua sắm } & $1-2$ lần & 43 & $26,7 \%$ \\
\hline & 3 -4 lần & 73 & $24,3 \%$ \\
\hline & $5-6$ lần & 61 & $20,3 \%$ \\
\hline & Trên 6 lần & 86 & $28,7 \%$ \\
\hline \multirow{4}{*}{ Thời gian sử dụng FB } & $<30$ phút & 23 & $7,7 \%$ \\
\hline & 30 phút đến 1 tiếng & 99 & $33 \%$ \\
\hline & 1 đến 2 tiếng & 92 & $30,7 \%$ \\
\hline & Trên 2 tiếng & 86 & $28,7 \%$ \\
\hline \multirow{6}{*}{ Mục đích sử dụng FB } & Mua sắm & 118 & $39,3 \%$ \\
\hline & Xem tin tức & 207 & $69,3 \%$ \\
\hline & Giải trí & 136 & $47,3 \%$ \\
\hline & Trò truyện & 194 & $64,7 \%$ \\
\hline & Công việc & 92 & $29 \%$ \\
\hline & Tìm kiếm bạn bè & 42 & $14 \%$ \\
\hline \multirow{8}{*}{$\begin{array}{l}\text { Lý do chọn FB là kênh } \\
\text { mua sắm }\end{array}$} & Uy tín & 22 & $2,7 \%$ \\
\hline & Chất lượng & 23 & $2,9 \%$ \\
\hline & Dịch vụ tốt & 48 & $6 \%$ \\
\hline & Dễ tìm kiếm & 212 & $26,4 \%$ \\
\hline & Nhiều mặt hàng & 198 & $24,7 \%$ \\
\hline & Tương tác tốt & 185 & $23 \%$ \\
\hline & Biết rõ người bán & 66 & $8,2 \%$ \\
\hline & Giá cả tốt & 49 & $6,1 \%$ \\
\hline
\end{tabular}

Bảng 2: diễn giải thang đo

\begin{tabular}{|l|l|}
\hline & Niềm tin đối với sản phẩm \\
\hline NTSP1 & sản phẩm được bán trên Facebook hoàn toàn chất lượng \\
\hline NTSP2 & sản phẩm được bán trên Facebook đúng với hình ảnh mà người bán quảng bá \\
\hline NTSP3 & sản phẩm được bán trên Facebook có nguồn gốc xuất xứ rõ rang \\
\hline NTSP4 & những sản phẩm được mua trên Facebook hoàn toàn hữu ích \\
\hline
\end{tabular}




\begin{tabular}{|c|c|}
\hline & Niềm tin về những ảnh hưởng của môi trường xã hội \\
\hline NTXH1 & Những nhân xét của khách hàng trên FB ảnh hưởng đến việc mua hàng \\
\hline NTXH2 & Ý kiến của những người quan trong ảnh hưởng đến viêc mua sắm quần áo trên Facebook \\
\hline NTXH3 & Mọi người mong đợi sử dụng những sản phẩm đó \\
\hline \multirow[t]{2}{*}{ NTXH4 } & tham khảo ý kiến của những khách hàng đã từng mua quần áo trên Facebook \\
\hline & Live stream-Truyền tải trực tiếp \\
\hline LV1 & $\begin{array}{l}\text { Khi livestream thì khu vực được giới thiệu có ảnh hưởng đến đánh giá chung về uy tin của } \\
\text { người bán }\end{array}$ \\
\hline LV2 & $\begin{array}{l}\text { Khi livestream thì sẽ được cảm nhận được hình ảnh thật hơn về sản phẩm so với những } \\
\text { hình ảnh được quảng cáo }\end{array}$ \\
\hline LV3 & Khi livestream thì người mẫu hoặc người giới thiệu là quan trọng \\
\hline \multirow[t]{2}{*}{ LV4 } & Thời điểm live stream là rất quan trọng \\
\hline & Uy tín người bán \\
\hline UTNB1 & Uy tín của người bán được thể hiện qua lượt like và theo dõi \\
\hline UTNB2 & Trang bán hàng uy tín khi được nhiều khách hàng đánh giá tốt và xếp hạng cao \\
\hline UTNB3 & $\begin{array}{l}\text { Uy tín của người bán thể hiện qua cách trả lời tin nhắn hoặc comment trên Facebook có } \\
\text { nhiệt tình hay không }\end{array}$ \\
\hline \multirow[t]{2}{*}{ UTNB4 } & $\begin{array}{l}\text { thường tìm kiếm thông tin của người bán hàng trên Facebook qua các công cụ tìm kiếm( } \\
\text { google, bing, ....) }\end{array}$ \\
\hline & Chính sách hoàn trả \\
\hline CSHT1 & Sản phẩm có thể hoàn trả nếu như không đúng cam kết của người bán \\
\hline CSHT2 & cảm thấy rắc rối với những chính sách hoàn trả của người bán hàng trên FB \\
\hline \multirow[t]{2}{*}{ CSHT3 } & $\begin{array}{l}\text { có quyền yêu cầu không nhận hàng nếu như quá thời gian nhận hàng so với cam kết ban } \\
\text { đầu }\end{array}$ \\
\hline & Sự thuận tiện \\
\hline STT1 & có thể dễ dàng nắm bắt được cách thức đặt hàng \\
\hline STT2 & $\begin{array}{l}\text { mua hàng trên mạng xã hội Facebook tiện lợi hơn so với các mạng xã hội hay hình thức } \\
\text { khác }\end{array}$ \\
\hline STT3 & có thể tìm kiếm dễ dàng được sản phẩm mình cần trên Facebook \\
\hline \multirow[t]{2}{*}{ STT4 } & thoải mái lựa quần áo trên Fb dù bất kì ở nơi đâu chỉ với smartphone \\
\hline & Hành vi mua sắm qua $\mathrm{FB}$ của người tiêu dùng \\
\hline H1 & hài lòng với việc mua quần áo thời trang trên Facebook \\
\hline $\mathrm{H} 2$ & sẽ giới thiệu những người khác mua quần áo thời trang trên Facebook \\
\hline $\mathrm{H} 3$ & tiếp tục mua quần áo thời trang trên Facebook \\
\hline
\end{tabular}

\subsection{Phân tích Cronbach's Anlpha và các biến phụ thuộc}

Trong nghiên cứu này tác giả sử dụng hệ số đo lường độ tin cậy của thang đo Cronbach's Alpha có giá trị lớn hơn hoặc bằng 0,6 và hệ số tương quan biến tổng Corrected Item-Total Correlation có giá trị lớn hơn hoặc bằng 0,3 thì thang đo mới đủ độ tin cậy để thực hiện các bước phân tích tiếp theo

\begin{tabular}{|l|l|l|l|l|}
\hline STT & Nhân tố & Số biến quan sát & Cronbach's Alpha & $\begin{array}{l}\text { Biến quan sát } \\
\text { bị loại }\end{array}$ \\
\hline 1 & Niềm tin đối với sản phẩm & 4 & 0,838 & \\
\hline 2 & $\begin{array}{l}\text { Niềm tin về những ảnh hưởng } \\
\text { của môi trường xung quanh }\end{array}$ & 4 & 0,746 & NTXH 2 \\
\hline 3 & Chính sách hoàn trả & 3 & 0,815 & \\
\hline 4 & Uy tín người bán & 4 & 0,794 & \\
\hline 5 & $\begin{array}{l}\text { Livestream- truyền tải trực } \\
\text { tuyến }\end{array}$ & 4 & 0,801 & \\
\hline 6 & Sự thuận tiện & 4 & 0,832 & \\
\hline 7 & Hành vi mua sắm & 3 & 0,715 & \\
\hline
\end{tabular}


Sau khi phân tích thì hệ số tương quan của biến quan sát NTXH2 là $0,262<0,3$ nên ta loại biến này và chạy lại lần 2. Sau khi phân tích hệ số Cronbach's Alpha của thang đo lần 2, dựa vào kết quả bảng thống cho thấy hệ số Cronbach's Alpha tổng tổng thể của thang đo giá trị là $0,746>0,6$ và hệ số tương quan biến tổng của 3 biến quan sát đều có giá trị $\geq 0,3$ nên thang đo đủ tin độ tin cậy để thực hiện các phân tích tiếp theo.

\subsection{Phân tích nhân tố khám phá EFA}

Tóm tắt kết quả phân tích nhân tố (EFA):

Biến UTNB1 tải lên 2 lần nên ta chạy lại lần 2.Kết quả phân tích nhân tố cho thấy các biến có trọng số tải nhân tố (Factor loading) đều lớn hơn 0,5 nên các biến quan sát đều quan trọng trong các nhân tố. Hệ số $\mathrm{KMO}=0,742>0,5$ nên phân tích EFA phù hợp với dữ liệu. Kiểm định Bartlett's test có mức ý nghĩa 0,000 $<0,05$, do vậy các biến quan sát có tương quan với nhau xét trên phạm vi tổng thể. Giá trị Eigenvalue $=$ $1,776>1$ đạt yêu cầu, 21 biến quan sát được nhóm lại thành 6 nhân tố. Phương sai trích được bằng $68,167 \%$, cho biết 6 nhân tố giải thích được $68,167 \%$ biến thiên của dữ liêu nghiên cứu. Ngoài ra, 6 nhân tố được hình thành sau khi phân tích EFA đều có giá trị Cronbach's Alpha $>0,6$ nên 6 thang đo này đạt yêu cầu khi phân tích ở các bước tiếp theo

Kết quả phân tích nhân tố cho thấy các biến độc lập và biến phụ thuộc trong mô hình nghiên cứu đều đạt giá trị hội tụ và giá trị phân biệt chấp nhận được, phân tích EFA là thích hợp với dữ liệu nghiên cứu. Qua phân tích nhân tố $\mathrm{EFA}$, các biến quan sát trong các nhân tố đều đo lường độ chính xác của các khái niệm mô hình nghiên cứu, 6 nhân tố được hình thành đều thoả mãn các tiêu chí kiểm định độ tin cậy và độ chính xác của thang đo. Như vậy, từ 6 nhân tố của mô hình nghiên cứu đề xuất ban đầu, chỉ còn 6 nhân tố với 21 biến quan sát của nhân tố độc lập và 3 biến quan sát của nhân tố phụ thuộc. Do đó, mô hình nghiên cứu đã đề xuất cần được hiệu chỉnh.

\begin{tabular}{|c|c|c|c|}
\hline Các tiêu chí & Biến đâc lân & Biến nhu thû̂c & Ý nohĩa \\
\hline Hệ số KMO & 0,742 & 0,622 & \multirow{2}{*}{$\begin{array}{l}\text { Hệ số KMO trong khoảng }(0,5-1) \text { và sig. } \\
=0,000<0,05 \text { cho thấy các biến quan sát } \\
\text { trong tồng thể có mối tương quan với } \\
\text { nhau và phân tích nhân tố EFA là phù hợp }\end{array}$} \\
\hline Sig. & 0,0000 & 0,0000 & \\
\hline Eigenvalues & 1,776 & 1,922 & \multirow{2}{*}{$\begin{array}{l}\text { Eigenvalues }>1 \text { và phương sai trích } \geq \\
50 \% \text {, kết quả thang đo các thành phần đạt } \\
\text { yêu cầu }\end{array}$} \\
\hline Phương sai trích & $68,167 \%$ & $64,064 \%$ & \\
\hline $\begin{array}{c}\text { Tỉ lệ quy mô mẫu và } \\
\text { biến quan sát }\end{array}$ & $11,5: 1$ & & \\
\hline
\end{tabular}

\subsection{Phân tích hồi quy}

\begin{tabular}{|c|c|c|c|c|c|c|c|c|}
\hline \multicolumn{9}{|c|}{ Bảng 4: Phân tích hôi quy } \\
\hline & \multirow[t]{2}{*}{ Model } & \multicolumn{2}{|c|}{$\begin{array}{l}\text { Hệ số hồi quy } \\
\text { chưa chuẩn hóa }\end{array}$} & \multirow{2}{*}{$\begin{array}{c}\text { Hệ số } \\
\text { hồi } \\
\text { quy } \\
\text { chuẩn } \\
\text { hóa } \\
\text { Beta }\end{array}$} & \multirow[t]{2}{*}{$\mathbf{t}$} & \multirow[t]{2}{*}{ Sig. } & \multicolumn{2}{|c|}{$\begin{array}{c}\text { Thống kê đa cộng } \\
\text { tuyến }\end{array}$} \\
\hline & & B & $\begin{array}{l}\text { Sai số } \\
\text { chuẩn }\end{array}$ & & & & $\begin{array}{l}\text { Độ chấp } \\
\text { nhận }\end{array}$ & VIF \\
\hline \multirow[t]{7}{*}{1} & (hằng số) & -245 &, 154 & & $-1,595$ &, 112 & & \\
\hline & X1_NTSP. & ,160 & ,023 & ,221 & 6,944 & ,000 & 984 & 1,016 \\
\hline & X2_STT1 &, 057 &, 021 &, 088 & 2,762 & ,006 & ,972 & 1,029 \\
\hline & X3_LV & ,333 & , 021 &, 495 & 15,593 & ,000 & ,990 & 1,011 \\
\hline & X4_CSHT. & ,311 & ,019 &, 512 & 16,165 & ,000 & ,995 & 1,005 \\
\hline & X5_UTNB. & ,168 & ,020 & 270 & 8,426 & 000 & ,974 & 1,027 \\
\hline & X6_NTXH. & ,046 & ,022 & 068 & 2,111 & 036 & ,964 & 1,037 \\
\hline
\end{tabular}


Như vậy kết quả hồi quy cho thấy rằng các giá trị Sig. của các nhân tố đều nhỏ hơn 0,05 và 6 giả thuyết đưa ra ban đầu là H1, H2, H3, H4, H5, H6 đều được chấp nhận. Như vậy các biến độc lập tác động thuận chiều đến hành vi mua sắm trực tuyến trên Facebook của người tiêu dùng .

Hồi quy không có nhân tố nào bị loại bỏ do Sig kiểm định của từng biến độc lập đều nhỏ hơn 0,05 .

Hệ số VIF các biến độc lập đều nhỏ hơn 10, như vậy không có đa cộng tuyến xảy ra.

Hệ số Durbin-Watson $(\mathrm{d})=1,973$ nên không có hiện tượng tự tương quan giữa các phần dư trong mô hình, mô hình nghiên cứu có ý nghĩa thống kê.

Đồng thời các hệ số Beta đều lớn hơn 0 cho thấy các biến độc lập tác động thuận chiều với biến phụ thuộc Phương trình hồi quy chuẩn hóa:

\section{$Y=0,512 C S H T+0,495 \mathrm{LV}+0,270 \mathrm{UTNB}+0,221 \mathrm{NTSP}+0,88 \mathrm{STT}+0,68 \mathrm{NTXH}$}

Phần phân tích thống kê mô tả đã cho thấy được những đặc tính nhân khẩu học nổi bật liên quan đến tần suất mua hàng trực tuyến trên Facebook, thời gian mua hàng, thời gian online Facebook, mục đích sử dụng Facebook, thu nhập, giới tính, độ tuổi, trình độ học vấn, nghề nghiệp của những người tiêu dùng đã từng mua mua sắm trực tuyến trên Facebook về quần áo thời trang.

Kết quả kiểm định cho thấy không có sự khác biệt về hành vi mua sắm trực tuyến trên Facebook của người tiêu dùng giữa các nhóm khách hàng có sự khác nhau về giới tính, nghề nghiệp. Và các nhóm khách hàng về thu nhập, độ tuổi, trình độ học vấn thì có sự khác biệt về hành vi mua sắm trực tuyến trên Facebook về quần áo thời trang.

Hành vi mua sắm trực tuyến trên Facebook của người tiêu dùng về quần áo thời trang được kiểm định là hoàn toàn chịu tác động bới các nhân tố: Niềm tin sản phẩm, sự thuận tiện, livestream-truyền tải trực tuyến, chính sách hoàn trả, uy tín người bán, niềm tin xã hội.

Kết quả phân tích hồi quy cho thấy biến phụ thuộc Y (hành vi mua sắm trực tuyến trên Facebook của người tiêu dùng về quần áo thời trang) chịu sự tác động của các biến độc lập Xi bao gốm các biến: Niềm tin sản phẩm, sự thuận tiện, livestreamt-truyền tải trực tuyến, chính sách hoàn trả, uy tín người bán, niềm tin xã hội

\section{KẾT LUẬn VÀ HÀM Ý QUẢN TRI \\ 5.1 Kết luận}

Mô hình đề xuất 6 nhân tố ảnh hưởng đến hành vi mua sắm trực tuyến trên Facebook của người tiêu dùng bao gồm: Niềm tin sản phẩm, niềm tin xã hội, Livestream-truyền tải trực tuyến, chính sách hoàn trả, uy tín người bán, sự thuận tiện với 26 biến quan sát.

Sau khi đánh giá độ tin cậy của thang đo và phân tích nhân tố, biến quan sát NTXH2 (Ý kiến của những người quan trọng ảnh hưởng đến việc mua sắm quần áo trên Facebook của $\mathrm{A} / \mathrm{C}$ ), UTNB1(Uy tín của người bán được thể hiện qua lượt like và theo dõi) bị loại tương quan biến tổng nhỏ hơn 0.3 và truyền tải nhân tố hai lần.

Kết quả của hồi quy đa biến đã xác định hành vi mua sắm trực tuyến trên Facebook của người tiêu dùng về quần áo thời trang ảnh hưởng bởi 6 nhân tố và sắp xếp theo thứ tự mạnh nhất đến yếu tố yếu dần là: Chính sách hoàn trả, livestream-truyền tải trực tuyến, uy tín người bán, niềm tin sản phẩm, sự thuận tiền và cuối cùng là niềm tin xã hội.

Ngoài ra kết quả kiểm định cho thấy không có sự khác biệt về hành vi mua sắm trực tuyến trên Facebook của người tiêu dùng giữa các nhóm khách hàng có sự khác nhau về giới tính, nghề nghiệp. Và các nhóm khách hàng về thu nhập, độ tuổi, trình độ học vấn thì có sự khác biệt về hành vi mua sắm trực tuyến trên Facebook về quần áo thời trang.

\subsection{Hàm ý quản trị}

Thông qua nghiên cứu này, các doanh nghiệp tổ chức cá nhân kinh doanh về quần áo thời trang sẽ hiểu rõ hơn về hành vi mua sắm của người tiêu dùng trên $\mathrm{FB}$ hiện nay, từ đó xây dựng chính sách quảng bá, bán hàng phù hợp nhằm nâng cao doanh thu cũng như hiệu quả trong kinh doanh.

\subsubsection{Chính sách hoàn trả}

Đưa ra chính sách hoàn trả hợp lý. Thống nhất về các điều khoản hoàn trả để tránh sự không rõ ràng hay mâu thuẫn giữa hai bên. Chẳng hạn như khi khách hàng nhận phải hàng hóa không đúng với mẫu mã, chất lượng khi người bán giới thiệu thì được quyền hoàn trả và không tốn bất kì chi phí nào cho lần đặt hàng này. Phải đảm bảo giải quyết nhanh gọn lẹ đối với các trường hợp khách hàng hoàn trả sản phẩm do lỗi doanh nghiệp, tổ chức, cá nhân và xử lý nhẹ nhàng với những trường hợp khách yêu cầu đổi trả sai chính 
sách ban đầu. Từ những việc này có thể giúp cho các doanh nghiệp, tổ chức, cá nhân kinh doanh về quần áo thời trang có được sự tín nhiệm cũng như không phải đắn đo về chính sách hoàn trả của mình.

\subsubsection{Livestream-Truyền tải trục tuyến}

Khi livestream thì cần chú ý tới khu vực xung quanh mình bán hàng, cần phải gọn gàng, chuyên nghiệp để có thể gây ấn tượng tốt cũng như thu hút lượt xem của người tiêu dùng. Hiện nay trên FB có rất nhiều bài bán hàng cho nên ấn tượng ban đầu cực kì quan trọng vì nó quyết định rằng người tiêu dùng có ở lại để xem tiếp những gì mình bán hay là chỉ lướt qua.

Kèm theo đó người mẫu quảng cáo phải phù hợp với những mặt hàng mình bán. Một số người tiêu dùng ở lại video đấy là do sự thu hút của người mẫu, người mẫu mặc đẹp thì kích thích sự thích thú cũng như nhu cầu được như người quảng cáo.

Kế tiếp người giới thiệu trực tuyến có giọng nói thu hút, truyền tải được tới người nghe sẽ là một lợi thế rất lớn.

Từ những vấn đề trên thì các doanh nghiệp, tổ chức, cá nhân nên có những chính sách thích hợp nhằm nâng cao khả năng bán hàng bằng video trên $\mathrm{FB}$ của mình.

\subsubsection{Uy tín người bán}

Sự tin tưởng là cốt lõi của một giao dịch trực tuyến. Trên thị trường hiện này, có rất nhiều trang bán hàng FB được lập ra, tính cạnh tranh ngày càng cao, vì vậy mà uy tín của doanh nghiệp, tổ chức, cá nhân càng cao thì sự lựa chọn của người tiêu dùng cũng tỉ lệ thuận. Những người trước khi có hành vi mua sắm trên FB thì họ đều tham khảo về mức uy tín của trang bán hàng của cá nhân, tổ chức hay doanh nghiệp đấy. Một trang bán hàng với mức đánh giá cao của khách hàng, nhiều thông tin tốt trên các công cụ tìm kiếm sẽ làm cho người tiêu dùng đấy có một sự an tâm nhất định trước khi quyết định mua sắm tại đây. Ngoài ra, sự phản hồi lại của doanh nghiệp, tổ chức, cá nhân đó khi khách hàng đặt câu hỏi về bên mình là một yếu tố cực kì quan trọng. Sự tương tác nhanh, chuyên nghiệp, nhiệt tình càng thúc đẩy nhanh chóng nhành vi của người khách hàng đấy.

Từ những vấn đề trên mà các doanh nghiệp, tổ chức, cá nhân đang bán hàng trên FB nên:

Có một trang bán hàng chuyên nghiệp, có một đội ngũ chăm sóc khách hàng, nhờ khách hàng đánh giá và xếp hạng trang bán hàng của mình nhằm nâng cao uy tín.

Đào tạo bộ phận chăm sóc khách hàng cũng như bán hàng một cách có bài bản.

Đầu tư chính sách quảng bá, quảng cáo trang bán hàng để nhiều khách hàng biết đến doanh nghiệp, tổ chức của mình.

\subsubsection{Niềm tin sản phẩm}

Người tiêu dùng vẫn chưa thực sự an tâm đối với chất lượng của quần áo mình mua trên Facebook. Tình trạng khi nhận hàng hóa không đúng với những hình ảnh hay chất lượng mà người bán ban đầu cam kết vần đang diển ra. Để giải quyết vấn đề này, thì các doanh nghiệp, tổ chức, cá nhân khi bán hàng qua FB phải đưa ra những hình ảnh thật, đảm bảo chất lượng về hàng hóa cho người tiêu dùng, cam kết những thông tin về sản phẩm mình bán là thật. Với nhiều sự cam kết và chính sách đảm bảo thì sẽ phần nào làm cho khách hàng của mình thực sự an tâm và hành vi mua sắm sẽ được nâng cao hơn.

\subsubsection{Sự thuận tiện}

Các doanh nghiệp, tổ chức, cá nhân phải nâng cao tính linh hoạt và sự đa dạng về cách thức quảng bá cũng như giới thiệu sản phẩm của mình. Phải đảm bảo cho người tiêu dùng có thể truy cập vào trang bán hàng, trang cá nhân bất kỳ thời gian và không gian nào bằng cách cải thiện trang bán hàng, sắp xếp hàng hóa cần bán theo thứ tự một cách hợp lý để người tiêu dùng tìm được những sản phẩm mình cần một cách nhanh chóng và thuận tiện. Bên canh đó do nhu cầu cũng như thị hiểu của người tiêu dùng ngày càng tăng cao và đa dạng vì vậy mà các tổ chức, doanh nghiệp, cá nhân cần không ngừng nâng cao, hoàn thiện phương thức bán hàng của mình nhằm tạo ra sự tiện lợi nhất cho người tiêu dùng.

5.2.6 Niềm tin về nhũng ảnh hương của môi truờng xã hộ

Các nghiên cứu thực nghiệm từ việc vận dùng hoặc mở rộng các mô hình lý thuyết hành động hợp lý cho thấy khách hàng đã từng hài lòng khi mua online sẽ dễ dàng mua lại ( Ribbink \& Van, 2004); người chưa mua sẽ có xu hướng đọc những bài nhận xét hoặc bình luận của người có kinh nghiệm để đưa ra quyết định của mình (Ellison, Steinfield, \& Lampe, 2007); một cá nhân trước khi quyết định mua sắm thường có xu hướng tham khảo thông tin từ các nhóm tham chiếu như bạn bè, đồng nghiệp, đặc biệt là những mặt hàng mang biểu tượng xã hội cao như sản phẩm may mặc thời trang ( Nandini \& Jeevanada, 2014). Vì vậy người 
quản lí trang bán hàng của doanh nghiệp, tổ chức, cá nhân đó nên tích cực trong việc nhờ người tiêu dùng đánh giá về uy tín cũng như chất lượng sau mỗi lần người tiêu dùng mua hàng. Để người tiêu dùng có thể đánh giá một cách nhanh chóng và tự nguyện thì người bán có thể đưa ra chính sách giảm giá 5-10\% cho mỗi lần đánh giá hoặc giới thiệu thành công cho bạn bè người thân của mình hoặc miễn phí phí vận chuyển...Từ những đánh giá như thế, khi người tiêu dùng trước khi mua quần áo thời trang, người tiêu dùng có thể tham khảo thông qua những bạn bè, đồng nghiệp đã từng mua hàng tại đó. Những phản hồi tích cực sẽ tác động rất nhiều đến hành vi mua hàng của người tiêu dùng và ngược lại. Có thể người bán đó rất tốt nhưng ít đánh giá thì cũng sẽ làm cho người tiêu dùng phải e ngại trước khi đưa ra quyết định của mình

\section{TÀI LIỆU THAM KHẢO}

Ajzen, I. (1985). From intentions to actions: A theory of planned behavior. In J.Kuhl \& J. Beckman (Eds.). Actioncontrol: From cognition to behavior, pp. 11- 39.

Ajzen, I. (1985). From intentions to actions: A theory of planned behavior. In J. Kuhl, \& J. Beckmann (Eds.). Springer series in social psychology, pp. 11-39.

Ajzen, I. (1991). "Theory of planned behavior". Organizaitonal Behavior and Human Decision Prcocesses. 50, pp. 179- 211.

Ajzen et al. (1992). A comparison of the Theory of Planned Behavior and the Theory of Reasoned Action. The Society for Personality and Social Psychology Inc, America

Bhatnagar, A., Misra, S. , \& Rao, H. R.. (2000). On Risk, Convenience and Internet Shopping Behavior, Communications of the ACM, 48 (2), pp. $98-105$

Liang, T. , \& Lai, H. (2000). Electronic store design and consumer choi ce: an empirical study, System Sciences Proceedings of 33rd International Conference in Hawaii..

Williams, Chris. (2000). "Facebook wins Manx battle for facebook.com". The Register.

Haubl, G \& Trifts, V. (2000). Consumer decision making in online shopping environment: The effects of interactive decision aids, Marketing Science, 19 (1), pp. 4- 21.

Dahiya Richa. (2000). "Impact of demographic factors of cunsumers on online shopping behavior: A study of cunsumers in India". International Journal of Engineering and Management Sciences 3.1, pp. 43- 52.

Werner, P. (2004). Reasoned Action and Planned Behavior, in Peterson, S. J., and Bredow, T. S. (Eds.); Middle range Theories: Application to Nursing Research; Lippincott Williams \& Wilkins, pp. 125- 147.

Sultan, Muhammad Umar, and Md Uddin. (2011). Consumers' Attitude towards Online Shopping: Factors influencing Gotland consumers to shop online. Diss. Gotland University.

Mohammad Hossein Moshref Javadi et al. (2012). An Analysis of Factors Affecting on Online Shopping Behavior of Consumers. Iran.

Shyh-Hwang Lee \& Hoang Thi Bich Ngoc. (2011). Điều tra các ý định mua sắm trục tuyến của sinh viên Việt Nam: mở rộng lý thuyết về hành vi dụ kiến.

Ngô Thị Minh Ngọc. (2015). Nghiên cúu các yếu tố ảnh huơơng đến hành vi mua sắm trục tuyến của nguời tiêu dùng trên địa bàn Tỉnh Thái Nguyên. Luận văn thạc sĩ kinh tế, Trường Đại Học Tài Chính Marketing.

Brandsvietnam.com. (2016). Truy xuất từ http://brandsvietnam.com/10147-Doanh-thu-quang-cao-truc-tuyen-o-VietNam-dang-chay-ve-tui-cua-Facebook-Google.

Phương Quyên. (2016). báo cáo thị truờng thời trang việt giai đoạn 2015-2016, Truy xuất từ https://maybanhang.net/2016/12/bao-cao-thi-truong-thoi-trang-viet/.

Ngày nhận bài: 23/02/2018

Ngày chấp nhận đăng: 20/03/2019 